Please cite this article as: Chamilothori K, Chinazzo G, Rodrigues J, Dan-Glauser E, Wienold J, Andersen M, Subjective and physiological responses to façade and sunlight pattern geometry in virtual reality, Building and Environment (2019), doi: https://doi.org/10.1016/j.buildenv.2019.01.009

\title{
Subjective and physiological responses to façade and sunlight pattern geometry in virtual reality
}

\author{
K. Chamilothori ${ }^{\mathrm{a},{ }^{*}}$, G. Chinazzo ${ }^{\mathrm{a}}$, J. Rodrigues ${ }^{\mathrm{b}}$, E. S. Dan-Glauser ${ }^{\mathrm{c}}$, J. Wienold ${ }^{\mathrm{a}}$, M. Andersen ${ }^{\mathrm{a}}$ \\ a Laboratory of Integrated Performance in Design (LIPID), School of Architecture, Civil and Environmental \\ Engineering, Ecole polytechnique fédérale de Lausanne (EPFL), 1015 Lausanne, Switzerland \\ ь Behavioral Genetics Laboratory (LGC), Brain Mind Institute, EPFL, 1015 Lausanne, Switzerland \\ c Cognitive and Affective Regulation Laboratory (CARLA), Institute of Psychology, University of Lausanne (UNIL), \\ 1015 Lausanne, Switzerland
}

\section{Highlights}

- Façade and sunlight pattern geometry affect participants' subjective responses.

- Façade and sunlight pattern geometry affect participants' mean heart rate change.

- Non-uniformly distributed openings led to lower mean heart rate than venetian blinds.

- Non-uniformly distributed openings were preferred to uniformly distributed openings.

- Space use scenarios influence how interesting and exciting a space is perceived.

\begin{abstract}
This study investigates the joint impact of façade geometry and associated sunlight patterns on occupant subjective perception and physiological responses through a novel experimental method coupling physically-based simulations shown in virtual reality with a wearable biometric device. A total of 72 subjects participated in a study combining three façade configurations of an equal aperture ratio with different scenarios of space use (a social or working context). The façade variations -a nonuniform distribution of openings ("Irregular"), a uniform distribution of openings ("Regular") and venetian blinds ("Blinds")- were applied to an interior scene with clear sky and direct sun penetration. Subjective evaluations (how pleasant, interesting, and exciting the space was perceived) and physiological responses (heart rate and skin conductance) were collected during exposure to façade variations, while a neutral scene was used to record baseline physiological responses. Results revealed
\end{abstract}


that façade and sunlight pattern geometry significantly influenced subjective responses for both context scenarios, while subsequent analyses showed differences mostly between the Irregular and Regular conditions, with the former being evaluated more positively. Façade and sunlight pattern geometry affected heart rate responses, but not skin conductance responses. In particular, participants showed a larger decrease in heart rate while exposed to the Irregular condition compared to the Blinds. Context scenarios influenced evaluations of interest and excitement. Findings are particularly relevant for applications in architecture and lighting, demonstrating that façade elements and their interaction with light can influence occupant subjective and physiological responses, and showcasing the potential of the presented method for investigating human perception.

\section{Keywords}

Façade, sunlight, pattern, perception, physiological response, virtual reality

\section{Introduction}

In the field of architecture, daylight has been widely recognized to have a profound impact on the way occupants experience space [1]-[4]. Nevertheless, current lighting design practices and daylighting metrics tend to restrict sunlight penetration in favor of visual comfort and energy savings, to avoid glare risk and overheating from the sun, respectively [5], [6]. Contrary to this tendency, the new European standard 'Daylight in buildings' requires a minimum number of hours during which a space receives direct sunlight [7]. The restriction of sunlight access has been criticized as a trend that produces monotonous luminous environments [8], [9], and is supported by evidence from lighting research, as non-uniformity in luminous conditions has been widely acknowledged to relate with impressions of interest [10].

The luminance variation in an interior space is inextricably linked to architectural elements that shape the way daylight enters the space, such as shading systems and window openings. However, there is a gap of research on how such façade characteristics and the resulting sunlight patterns can affect the occupant's experience of the indoor space. Although predictive models for the 
positive perceptual effects of contrast distribution in the user's field-of-view exist [11], we are lacking replicable knowledge about the impact of the characteristics of sunlight patterns and of the resulting light distribution in a space. Moreover, little attention has been given to the relation between the use of space and occupant perception, although it is acknowledged as an important factor in the occupants' evaluation of a luminous environment [12]-[14].

This article introduces a study which investigates the effect of the façade geometry and the resulting sunlight patterns on occupant perception through experiments in virtual reality (VR). In addition to participants' subjective evaluations, physiological responses of both heart rate and skin conductance were recorded as indicators of arousal and emotional response. The presented experimental study also addresses the effect of context on occupant responses, by using two scenarios of how the presented space might be used - a social and a working context- and exploring their impact on how occupants evaluate the luminous conditions. To the authors' knowledge, this is the first study investigating the impact of daylight and façade characteristics on both subjective and physiological responses, and addressing the effect of the use of space on the occupant's perception of luminous environments.

\section{State of the art}

A brief review of the literature on the positive perceptual effects of sunlight will help illustrate the relevance of the presented study. In this section, we will also present current challenges in experimental lighting research that hinder the advancement of knowledge on this topic, relating to both the independent and dependent variables commonly employed in relevant lighting research studies.

\subsection{Sunlight and positive effects on perception}

Research in the field of lighting has shown the positive perceptual effects of sunlight penetration on office workers, such as increased relaxation [15] and feeling of well-being [16]. These positive effects of sunlight have been suggested to increase glare tolerance [17]. Contrary to expectations, occupants of office environments chose to allow direct sunlight into the room when given the choice to control 
the blinds and create their preferred condition [18]. In the same vein, the contrast and luminance variation in the user's field of view, resulting from allowing sunlight access, have been repeatedly linked with the creation of interest both in real spaces [19], [20] and in virtual environments [21], [11]. Even though there is substantial evidence of the positive effects of sunlight access, we have limited knowledge on how the façade geometry and the spatial composition of the resulting sunlight patterns affect occupant perception and emotional state. Previous research has highlighted the importance of façade and sunlight pattern characteristics, such as complexity [22], [23] on visual perception, but has been limited either in the statistical analysis or the sample size of the study. In particular, a preliminary study by the authors showed that non-uniformly distributed façade openings and the resulting sunlight patterns were evaluated more positively than uniformly distributed openings [24], suggesting that the spatial composition of the façade and sunlight patterns necessitates further investigation.

Although researchers acknowledge the importance of luminance variation for a stimulating luminous environment, they note equally the difficulty of differentiating between satisfactory and unsatisfactory conditions, especially in cases of high luminance [18]. This complexity of distinguishing the positive and negative perceptual effects of direct sunlight brings forth the subject of context - i.e. of the use of space - and its influence on our appraisal of daylit environments. In a study where participants were asked to declare their seating preference for different scenarios of use of a daylit space, the findings showed that sunlight access was not welcome in the context of teamwork, but it was preferred in the context of both isolated work and relaxation [13]. In alignment with these findings, it is suggested that it is necessary to employ a specific context, rather than an abstract experimental setting, in order to investigate high-order perceptions such as interest [14].

\subsection{Challenges of experimental studies in daylighting research}

\subsubsection{Changing daylight conditions}

Conducting experiments in real environments poses a great challenge for researchers, being complex in both parameters and resources. These limitations are especially relevant when investigating human 
perception, which requires a large number of participants to ensure statistically robust results. Furthermore, experimental studies in daylight face the particular problem of an independent variable which changes over time due to daily and seasonal variations [25], [26].

Two-dimensional visualizations based on photographs [27], [26], [28] or computationally rendered images [29], [30] have been suggested as a promising medium for investigating the visual perception of daylit spaces. Nevertheless, a study comparing the influence of different types of visualizations on participant responses showed that the evaluations of specific attributes differed significantly from those in the real environment [31]. An interactive two-dimensional panoramic projection, where the user could navigate the scene, was the only studied visualization type able to replicate the evaluation of perceived pleasantness and distribution of light in the real space, showing the importance of interactivity in the virtual environment for visual perception [31].

The use of VR headsets has been suggested as a means to increase this interactivity and improve the perceptual accuracy of the virtual environment [32], [33]. Recent studies have shown that there were no significant differences in participant task performance [34] and in the subjective evaluations of a daylit environment [35] between real spaces and their representation in immersive VR, demonstrating the use of VR as a very promising research tool. In particular, in a study conducted by the authors, an experimental method combining immersive VR and scenes generated from physically-based renderings was tested in three areas - perceptual accuracy in comparison with a real environment, reported adverse physical symptoms of the users, and perceived presence of the users in VR - and was shown to be an adequate surrogate for experiments investigating the visual perception of real environments [35].

\subsubsection{Quantifying the effect of luminous conditions on occupants}

In the context of lighting research, the subjective appraisal of the studied environment - such as judgements that a space appears interesting - has been argued to be intertwined with emotion processes [10]. In this context, the work of Russell et al. [36], [37] is particularly relevant, showing through factor analysis that emotional responses to environments can be reliably represented with 
two dimensions, valence (pleasure) and arousal (activation), illustrated in Figure 1. Although it has been argued that additional dimensions, such as potency, are required to adequately represent the domain of emotions [38], the dimensions of pleasure and arousal have been identified as especially pertinent for use in lighting research [15].

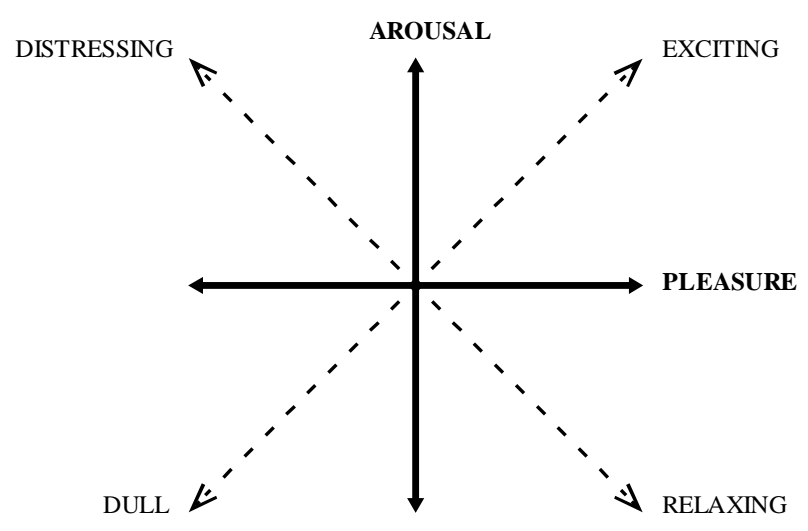

Figure 1. Russel's circumplex model of affect (adapted from Boubekri, 1991).

Existing research on the effect of façade geometry on occupant perception has used rating scales to assess the participants' appraisal of the environment [22]-[24]. Although this work is an important step in broadening our understanding of the topic, the validity of rating scales in quantifying the perception of a luminous environment has been questioned [39], [40]. An approach where subjective evaluations are coupled with an objective measure has been suggested instead as a more robust research instrument [41].

Measures of heart rate and skin conductance have been widely used as measures of arousal of the autonomic nervous system. Skin conductance has been shown to be sensitive to a wide range of phenomena, such as attention, activation, novelty and significance of a stimulus [42]. Heart rate, on the other hand, has been suggested to react in opposite directions when mental processing is required, where heart rate was shown to accelerate, or when attentional deployment is involved, where heart rate decelerates [43], and has thus been proposed as a more specific index of attention [44]. Similarly, low-arousal positive emotions, such as pleasure, have been associated with decelerated or unchanged heart rate, and unchanged skin conductance response [45]. Visual stimuli have been broadly used to elicit different emotional responses [46], [47]. The content of the visual stimulus, and particularly its 
valence - whether it is perceived as pleasant or unpleasant -, has been shown to affect the magnitude of both heart rate and skin conductance reaction [48]. Compared to the range of valence of visual stimuli used in the literature, inanimate objects - including scenes of interior spaces - would be considered neutral [49], and are thus expected to elicit physiological reactions of small magnitude.

Studies employing measures of heart rate and skin conductance to investigate the effect of virtual representations of the built environment show mixed results regarding their sensitivity [50][52] and suggest heart rate indices as more responsive for applications of environmental perception. However, in lighting research, where both measures have been used to study the physiological effects of different artificial lighting conditions, only skin conductance was significantly affected by the lighting conditions [44]. Following these findings, both heart rate and skin conductance indices seem promising for experimental studies that investigate the combined effect of building elements and lighting on occupants.

\subsection{Problem statement}

While the importance of daylight on our perception of space has been broadly acknowledged both in the field of architecture and in lighting research, there is a lack of established knowledge about its positive perceptual effects on occupants. In particular, little information is known about the impact of the spatial distribution of sunlight, and how a façade and the corresponding sunlight pattern can affect the experience of a space. Moreover, although considerable research in lighting has been devoted to investigations in working environments, less attention has been paid to different uses of a space, and to how the spatial context might influence the occupant's expectations and perception of a daylit space. The literature suggests two important challenges in current experimental methods used in lighting research, which might be contributing in the aforementioned gaps of knowledge: the changing lighting conditions in real environments, and the use of rating scales as the sole method of data collection. 
This article presents an experimental study which aims to provide substantial evidence on how the façade and the resulting sunlight pattern can jointly affect the individuals' experience of a space, taking into consideration different scenarios of use of space, while introducing and using experimental tools that address the identified limitations of experiments in lighting research. To this end, we investigate the joint impact of the façade and sunlight pattern geometry on occupants through an experimental study that combines the use of VR and the collection of both subjective (self-reported evaluations) and objective (physiological) participant responses. In particular, we investigate the following hypotheses:

1) The participants' perception of the space is influenced by the joint impact of the façade geometry and corresponding sunlight pattern in the virtual scene.

2) The participants' physiological responses are influenced by the joint impact of façade and sunlight pattern in the virtual scene.

3) A façade with a non-uniform - i.e. irregular - distribution of openings, along with the resulting sunlight pattern, will trigger interest in the virtual scene.

4) The participants' evaluation of the space will be influenced by the type of activity they expect to conduct in the presented virtual scene.

A total of 72 participants were exposed to immersive VR scenes of a daylit interior space with three façade variations of an equal aperture ratio: a non-uniform distribution of rectangular openings, a uniform distribution of the same openings, and venetian blinds, which will be referred to as "Irregular", "Regular" and "Blinds" façade variations, respectively. An additional neutral interior scene without access to daylight or view out was used in the beginning of the experiment to record baseline physiological responses in VR. Participants' subjective impressions - how pleasant, interesting, and exciting the space was perceived - and physiological responses - heart rate and skin conductance - were collected during their exposure to each façade variation. Moreover, two different 
context scenarios of the virtual environment - a working and a social context - were compared to one another to examine the perception of a luminous environment under different scenarios of use.

\section{Method}

\subsection{Experimental design}

In this study, we used a within-subject experimental design that included the within-subject factor façade geometry with three levels (Irregular, Regular, Blinds), and the between-subject factor context scenario with two levels (social or working context). The use of façade geometry, which is the main focus of this study, as a within-subject factor due to its advantage of eliminating the effect of variance between individual participants through the use of repeated measures, as each participant was exposed to all three façade variations. Before administering the verbal questionnaire, the between-subjects variable context was introduced by specifying the use of the presented scene - social or working activities - to the participant. Counterbalancing was used to avoid the introduction of confounding variables. In particular, experimental sessions were designed to counterbalance the order of stimulus presentation, the participants' gender, and the time of day of the experimental sessions (morning or afternoon) to account for possible effects of fatigue.

\subsection{Independent variables}

This study aims to investigate the combined effect of façade geometry and of the resulting sunlight pattern on participant responses. The studied façade variations include horizontal blinds ("Blinds"), used to provide a benchmark of a shading system that is commonly used in Europe, and a perforated façade with uniform ("Regular") and non-uniform ("Irregular") distribution of openings, used to investigate the effect of the spatial composition of façade openings and relating to characteristics of order [23] and complexity [22], [24] which have been identified as important in the literature. The three façade variations, shown in Figure 2, have an equal aperture ratio of 25\% (open to total window area), and differ either in the geometry of the aperture or in the spatial distribution of the openings on the façade. Regarding the geometry of the aperture, the "Blinds" variation differs from "Irregular" 
and "Regular", which have rectangular openings. The variation "Irregular" has an irregular distribution of openings, differing from the regular spatial distribution of the openings in the variations "Regular" and "Blinds". An overview of the shared and unique attributes between the façade variations is presented in Table 1. The combination of the three façade geometry variations and direct sun penetration produces the visual stimuli used in the present study. The simulation workflow for the creation of an immersive scene for each visual stimulus is described in section 3.4.

Although it is not treated as an independent variable, an additional interior scene was used in the beginning of the experiment to establish the participants' baseline physiological responses in virtual reality. As it is necessary to employ a neutral condition to record baseline measurements, this scene was generated from a high dynamic range (HDR) photograph of the experimental room from a participants' viewpoint. The HDR photograph was captured with automatic exposure bracketing with a Canon 70D camera and a $180^{\circ}$ Sigma $4.5 \mathrm{~mm}$ lens [53], [54], tone-mapped with the Reinhard02 tone-mapping algorithm [55] and gamma-corrected with a factor of 2.2. The resulting neutral scene, shown in Figure 2, has artificial light and no view access to match the actual visual conditions in the test room during the experimental session.
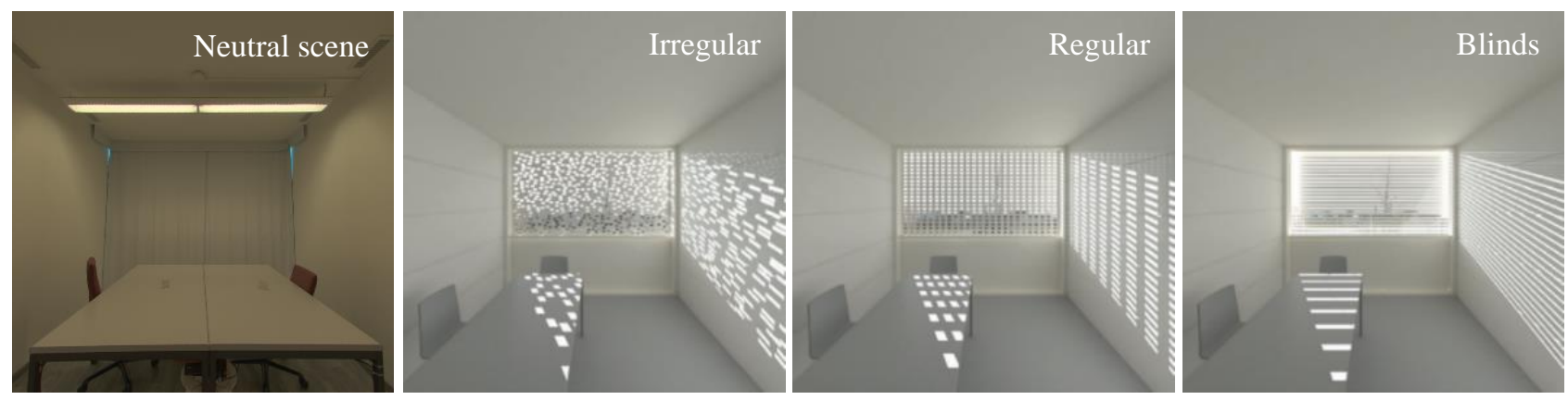

Figure 2. Front view of the neutral scene used for the recording of baseline physiological measures ("Neutral scene") and of the interior scene with façade variations ("Irregular", "Regular", "Blinds") that were used as independent variables in the study.

Table 1. Overview of the shared $(\checkmark)$ and unique $(X)$ attributes between the façade variations.

\begin{tabular}{lccc}
\hline Façade variation & Irregular & Regular & Blinds \\
Aperture ratio & $\checkmark$ & $\checkmark$ & $\checkmark$ \\
Geometry of aperture & $\checkmark$ & $\checkmark$ & X \\
Spatial distribution of openings & $\mathrm{X}$ & $\checkmark$ & $\checkmark$ \\
\hline
\end{tabular}




\subsection{Dependent variables}

\subsubsection{Subjective responses}

While exposed to each immersive scene, participants were asked to respond to a verbal questionnaire, and evaluated their impressions of how pleasant, interesting, and exciting the space was perceived on a scale from 1 (not at all) to 10 (very). The questions were asked in randomized order, and can be found in Table A of the Appendix. The attributes pleasant and exciting correspond to the first quadrant of the two-dimensional space of Russel's circumplex model of affect (shown in Figure 1), while interesting stems from existing literature in lighting research that relates luminous conditions with interest [10], [19]-[21].

\subsubsection{Physiological responses}

Heart rate (HR) and skin conductance (SC) was measured throughout each experimental session while the participant was immersed in VR. The mean HR response to each visual stimulus was calculated for the first 28 seconds after stimulus exposure, which was the largest common exposure time across stimuli presentation. This duration encompasses initial orienting responses that have classically been observed when participants were exposed to moving scenes [56], [57], as well as recovery from orientation and secondary emotional reaction to presented virtual environments [58], [59], including when seen in a VR setting [60]. Using the Ledalab V.3.4.9 toolbox in MATLAB, the raw SC data were separated into phasic and tonic components using the continuous decomposition analysis model [61] with default settings and four sets of initial values in the optimization. The skin conductance response (SCR), a measure which has been related to attention and arousal [42], was extracted for a response window from 1 to 4 seconds after each stimulus presentation, using a minimum amplitude threshold of $0.01 \mu \mathrm{S}$. Both the response window and the minimum amplitude threshold follow methodological recommendations for [42], [62].

Experimental changes in HR and SCR indices were obtained by subtracting their respective baseline values, to allow for comparison between participants. The baseline was measured during the presentation of the neutral scene in VR, using the response window that corresponded to each 
physiological indicator. The baseline subtracted indices will henceforth be indicated with " $\Delta$ " $-\Delta H R$ and $\triangle \mathrm{SCR}$ for the mean HR and mean SCR, respectively - and will be referred to as "change" to differentiate them from the measured values during the stimulus presentation.

\subsection{Simulation workflow}

\subsubsection{Rendering parameters}

The three façade geometries were applied to the digital model of the room where the experiment took place. Using an existing 3D model of the room (courtesy of the LESO Laboratory, EPFL [63]), and the Rhinoceros modelling software (https://www.rhino3d.com/), three variations of the test room were generated, each with a different shading system applied to the south façade. The room has mainly achromatic surfaces, windows on the south façade, and is equipped with a table and two chairs. Each test room variation was exported and rendered with Radiance (http://www.radianceonline.org/), an extensively validated physically-based lighting simulation tool [64], through the DIVA-for-Rhino toolbar [65]. A Konica Minolta CM-600d spectrophotometer was used to measure the color and specularity of the surfaces in the real room, which were translated to Radiance material properties used in the simulation (shown in Table B in the Appendix). The material of the existing venetian blinds in the experimental room was used for all three façade variations. Three scenes were rendered, one for each façade variation, with the same clear sky type generated with the gendaylit command. The Radiance rendering parameters and details of the sky description are summarized in

\section{Table 2.}

Table 2. Radiance rendering parameters

\begin{tabular}{|c|c|c|c|c|c|c|c|c|c|c|c|}
\hline -vs & $-\mathrm{vl}$ & $-a b$ & $-s$ & -st & $-1 v$ & $-\mathrm{ad}$ & -as & -aa & $-a r$ & $-p s$ & $-\mathrm{pj}$ \\
\hline 0 & 0 & 5 & 1 & 0 & 0.00001 & 20000 & 10000 & 0.05 & 512 & 0 & 0 \\
\hline \multicolumn{12}{|c|}{ Radiance sky generated with gendaylit: gendaylit 11259.5 -a 46.5190 -o -6.663 -m 0 -W 493.693 .3} \\
\hline \multicolumn{12}{|c|}{ Resolution: 3600 x 3600 (scaled down to $1200 \times 1200$ using pfilt) } \\
\hline \multicolumn{12}{|c|}{ Rendering time: 16 hours for all six view directions } \\
\hline
\end{tabular}

The exposure of the high dynamic range renderings from Radiance was adjusted by applying a uniform exposure multiplier with pfilt, and the resulting images were then converted to low dynamic range BMP files using $r a \_b m p$ with a gamma correction factor of 2.2. 


\subsubsection{Generation of immersive VR scenes}

The renderings that were obtained from Radiance consisted of two cubemap projections for each scene, generated from the viewpoint of each eye of a person looking towards the main view direction of the scene ( Figure 3a). These projections were used to generate a scene for VR ( Figure $\mathbf{3 b}$ ) following a workflow for creating immersive VR scenes from physically-based renderings, developed by the authors and described in [35]. The resulting scene is experienced in VR as a fully immersive $360^{\circ}$ environment seen from a static viewpoint, as illustrated in Figure 3c.

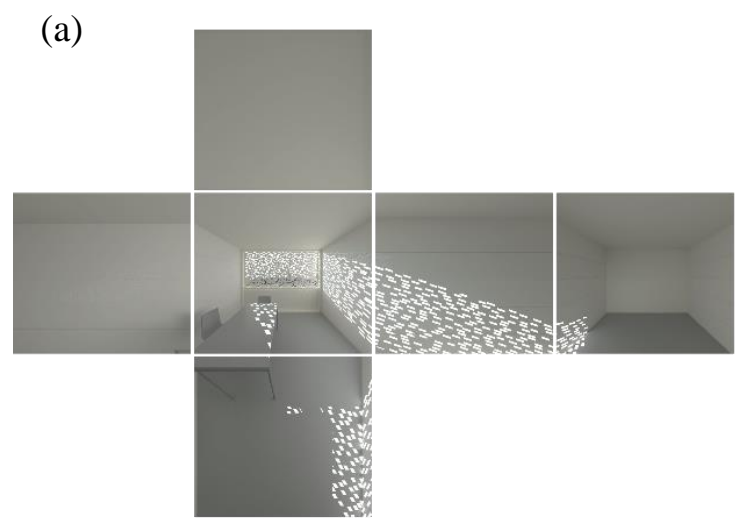

(b)

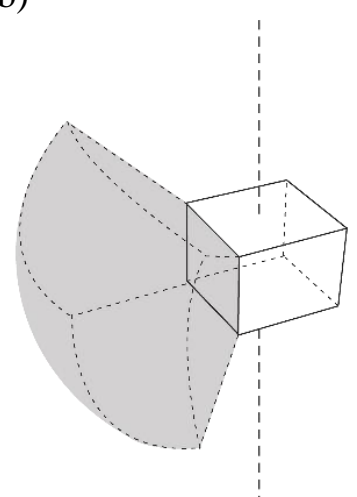

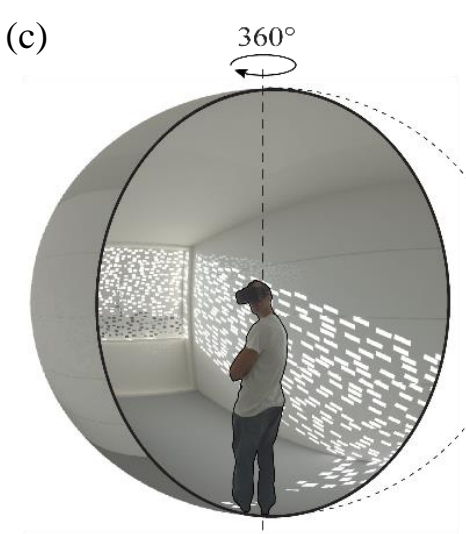

Figure 3. Example of the cubemap renderings for the right eye projection (a), and illustration of the world-to-cubemap projection (b) and the user's freedom of movement in the immersive scene (c).

The average RGB values of the cubemap projection, as well as the vertical illuminance at the center of each VR lens from the viewpoint of a participant were measured for each façade variation, to provide a measure of similarity between the scenes. The RGB values were transformed to the $\mathrm{L}^{*} \mathrm{a} * \mathrm{~b}$ color space to calculate the color difference $\Delta E^{*}{ }_{a b}$. The maximum $\Delta E^{*}{ }_{a b}$ between scenes was found to be equal to 0.83 , well below the threshold of 2.3 for color differences that are noticeable by the human eye [66]. Similarly, the three scenes differ between them in vertical illuminance with a maximum factor of 1.14 , below the threshold of 1.5 which represents the smallest significant difference for a just noticeable change in illuminance [67]. 


\subsection{Equipment}

The VR headset used in the study was an Oculus Rift CV1. The maximum luminance of the device measured at the lens was $80 \mathrm{~cd} / \mathrm{m} 2$, due to current technical limitations. The field of view of the device is $110^{\circ}$, while the display resolution is $1080 \times 1200$ pixels per eye with a $90 \mathrm{~Hz}$ refresh rate.

An Empatica E4 wristband [68], [69], worn on the left hand of the participant, was used to record HR and SC responses. For both SC and HR, physiological responses recorded with the Empatica E4 bracelet showed no significant differences when participants were exposed in physical environments and their $360^{\circ}$ representation in virtual reality [70]. The wristband uses a photoplethysmography sensor on the dorsal (outer) wrist that measures blood volume pulse data with a sampling rate of $64 \mathrm{~Hz}$ to compute HR. The Empatica E4 has been found to adequately measure HR data when tested against traditionally used electrocardiogram-based HR responses [69], with higher accuracy when the participant's movement is limited [71], as is the case in the present study. SC was measured through 2 ventral (inner) wrist electrodes of an $8 \mathrm{~mm}$ diameter contact area with a sampling rate of $4 \mathrm{~Hz}$. The device uses dry electrodes to measure SC, a method which has been found to be a valid and user-friendly alternative to traditionally used palmar electrodes [72] but leads to lower absolute measured values.

\subsection{Participants}

A total of 72 participants (36 women and 36 men) took part in the study, and were selected based on eligibility criteria of full color vision, a generally healthy condition, and English language proficiency of at minima $\mathrm{C} 1$ level. The participant age was limited to a range of 18 to 32 years (mean age: 25.9, $\mathrm{SD}=3$ years). This restricted age range was employed to ensure a homogeneous sample and to limit age related differences which have been found in the subjective perception of lighting conditions [73], [74], as well as in cardiac and electrodermal reactivity to affective stimuli [75]-[77]. 


\subsection{Experimental protocol}

The study was conducted in individual experimental sessions of 30 minutes, over a period of seven non-consecutive days in the summer. Air temperature was measured in each session (mean air temperature $=26.5^{\circ} \mathrm{C}, \mathrm{SD}=3^{\circ} \mathrm{C}$ ).

At the beginning of each session, participants were asked to read information about the study and sign a consent form. After consenting to participate in the study, participants wore the Empatica E4 wristband and responded to a series of demographic questions. They were then instructed to wear the VR headset and were guided in adjusting the headset's fit using a training scene. Participants were seated and were asked to limit their hand movements. Following this step, they were shown the experimental room from their viewpoint - a neutral interior scene, with artificial light and no view access - which was used to record baseline measurements. This scene was solely used to measure the participants' physiological responses to a neutral stimulus when wearing the VR headset in a resting state, and was not evaluated by the participants. The three façade geometry variations were subsequently shown one at a time in randomized order, and exposure to each scene was self-paced in order to minimize participant fatigue. For each condition of façade geometry, participants were instructed to take their time and explore the scene, and to inform the researcher when they were ready to verbally evaluate their perceptual impressions of the presented scene. The resulting mean exposure time to one scene was 68 seconds, while the largest common exposure time to one scene across all three façade variations was 28 seconds. The context scenario of the virtual environment for each experimental session was specified before starting the verbal questionnaire in each scene, by indicating to the participant to "Imagine you have a discussion with friends in this space" or "Imagine this is your office" for the social or working context, respectively.

This research was approved by the EPFL Human Research Ethics Committee and complied with the tenets of the Declaration of Helsinki. All participants provided written informed consent prior to the study and were compensated for their participation. 


\subsection{Data analysis}

\subsubsection{Dataset validity}

After visual inspection of the raw SC data, 6 problematic cases of non-responders [42] and 5 cases of sudden or intermittent signal breaks were identified and removed. The raw HR data was inspected for cases of values lower than $40 \mathrm{bpm}$ or higher than $200 \mathrm{bpm}$, or a difference higher than $3 \mathrm{bpm}$ between two consecutive measurements. Even though no such cases were identified, the sessions that were identified as problematic from the inspection of the SC data were also removed from the HR dataset, as a precaution for possible connectivity issues with the wristband. The removal of those cases, together with 3 other sessions where technical problems occurred, result in a dataset of 58 participants for the physiological responses, out of the initial sample of 72 . In addition, one of the sessions with technical problems had to be removed also from the dataset of the subjective responses, resulting in a total of 71 participants with valid self-reported data and a sample size of 35 and 36 participants for the social and working context scenario, respectively.

Altogether, the resulting sample sizes are 71 participants (36 men, 35 women) for the subjective responses and 58 participants (30 men, 28 women) for the physiological responses.

\subsubsection{Statistical analysis method}

A one-sample Kolmogorov-Smirnov test revealed that the data for all of the studied variables were not normally distributed, therefore non-parametric statistical tests were used. Main effects for the between-subjects factor of context were investigated with a Wilcoxon Rank-Sum test, while the main effects for the within-subject factor of façade geometry were investigated with a Friedman's one-way ANOVA for dependent samples [78]. In the case of a significant result in the Friedman's ANOVA, post-hoc tests were conducted for all pairwise comparisons using a Wilcoxon Signed-Ranks MatchedPairs test [78]. A Bonferroni-corrected significance level $\alpha$ of .0025 is used for the within-subject factor analyses of subjective responses to account for the 20 comparisons across all studied factors. Effect sizes $r$ [79] are reported for statistically significant effects in the pairwise comparisons, and they should be interpreted using the thresholds for a recommended minimum, moderate, and strong 
effect size of $.20, .50$ and .80 , respectively [80]. Lastly, the correlation between subjective and physiological responses is investigated by calculating the Spearman's correlation coefficient between the variables [81]. Statistical analyses were performed in MATLAB R2017a, using the Statistics and Machine Learning Toolbox.

\section{Results}

In the following section, we present the results of the statistical analyses for the main effects on participants' subjective and physiological responses, the correlation between subjective and physiological variables, as well as the possible effects of confounding factors on the findings.

\subsection{Subjective responses}

Although the main focus of the article is the effect of façade and sunlight pattern geometry on occupants, it is necessary to first investigate the impact of the spatial context scenarios on subjective responses, as this was a between-subject parameter.

A Wilcoxon Rank-Sum test comparing the two context scenarios and considering all three façade variations together revealed a statistically significant effect of context on perceived interest (rank sum=9840.5, p<0.01, r=.37), and perceived excitement (rank sum=9722, $\mathrm{p}<0.001, \mathrm{r}=.40$ ), but not on perceived pleasantness (rank sum $=1055.9, \mathrm{p}=0.13$ ). In particular, the space was perceived as less interesting and less exciting in the social context than in the work context (interest: median $_{\text {social }}=4$, median $_{\text {work }}=5$, excitement: median $_{\text {social }}=3$, median $_{\text {work }}=5$ ), which might reflect the discord between the participants' expectations regarding a space where they would socialize and the presented virtual environment. Although this finding demonstrates the influence of the use of space on how occupants evaluate a luminous environment, and is conducive to our fourth hypothesis for the evaluations of how interesting and exciting the space is perceived, further investigation on the effect of spatial context on the different façade and sunlight geometries exceed the scope of this article and will be addressed in a future publication. 
Following the result of a significant effect of spatial context on participant evaluations of interest and excitement, we investigate the impact of façade and sunlight geometry variation for each context scenario separately for these attributes.

A one-way Friedman's ANOVA for the three façade types showed a statistically significant effect of façade and sunlight pattern geometry on perceived interest and excitement for both the social $\left(\chi^{2}(2)_{\text {interesting }}=29.34\right.$, pinteresting $\left._{\text {in }}<.0001, \chi^{2}(2)_{\text {exciting }}=21.68, p_{\text {exciting }}<.0001\right)$ and the working context $\chi^{2}(2)_{\text {interesting }}=22.18$, pinteresting $\left.<.0001, \chi^{2}(2)_{\text {exciting }}=20.29, p_{\text {exciting }}<.0001\right)$. Perceived pleasantness, where the dataset contained data from both the social and the work context, was also significantly affected by the façade variation $\left(\chi^{2}(2)_{\text {pleasant }}=29.34, p_{\text {pleasant }}<.0001\right)$. These results support our first hypothesis, demonstrating that the façade geometry and the associated sunlight pattern jointly influence the participants' evaluations for all three studied attributes of how pleasant, interesting, and exciting the space was perceived.

In order to investigate further the differences in the evaluations of the three façade geometry variations, pairwise comparisons were conducted with a Wilcoxon Signed-Ranks Matched-Pairs test for all façade variations, for both the social and the work context, in the case of interest and excitement, and for the two context scenarios treated together, in the case of pleasantness. 


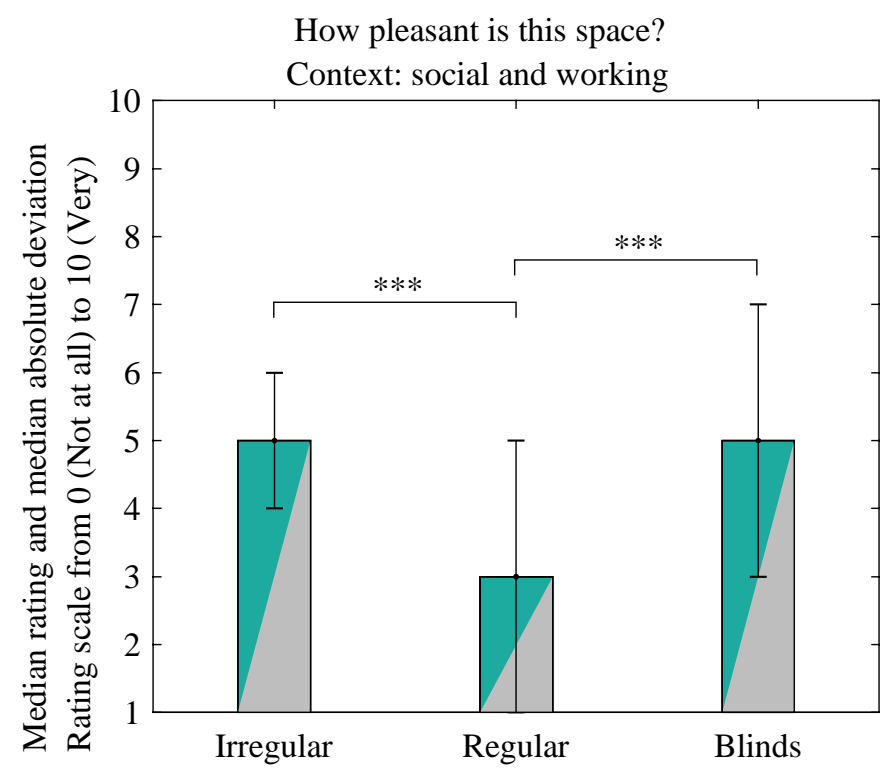

Datasets used:

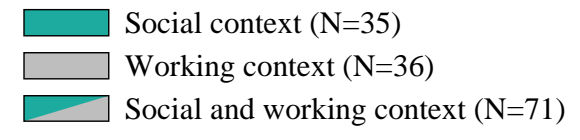

Significance level:

$* * *=\mathrm{p}<.001$

How interesting is this space?

How interesting is this space?
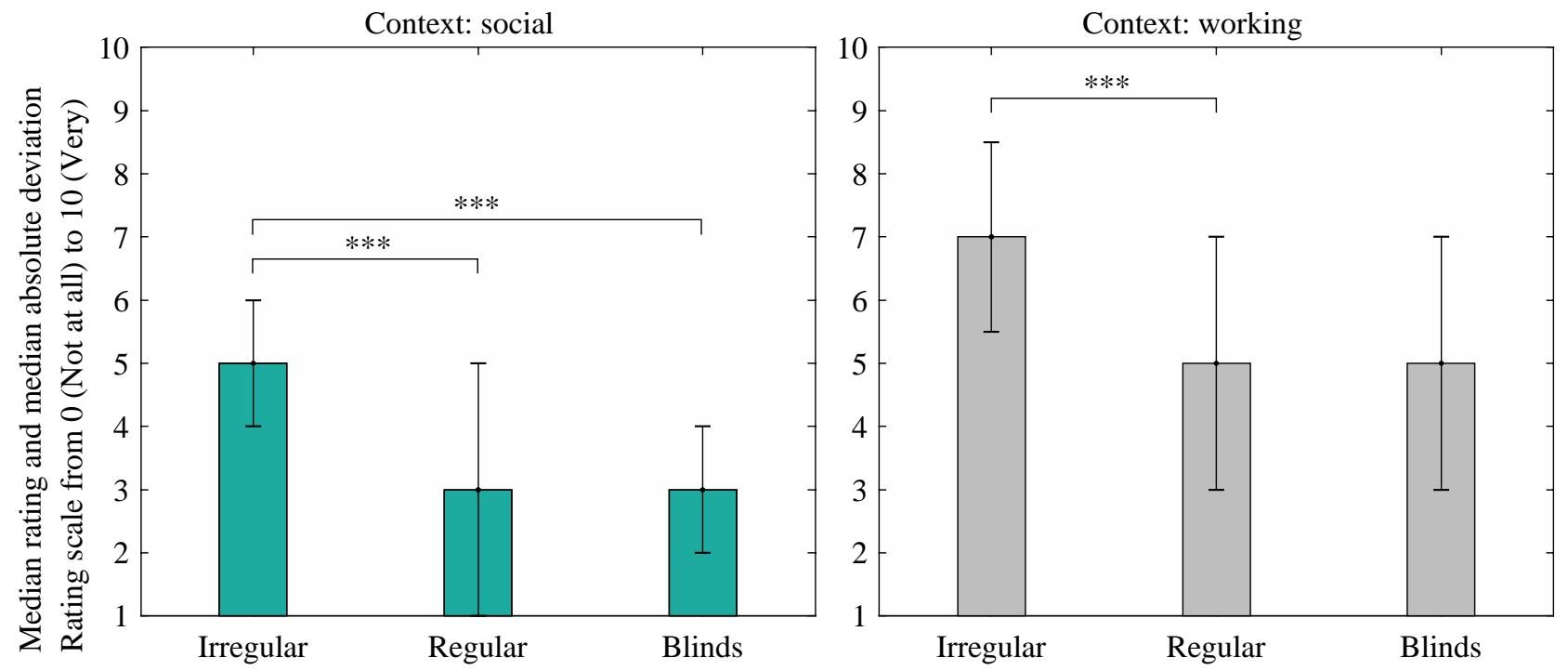

How exciting is this space?
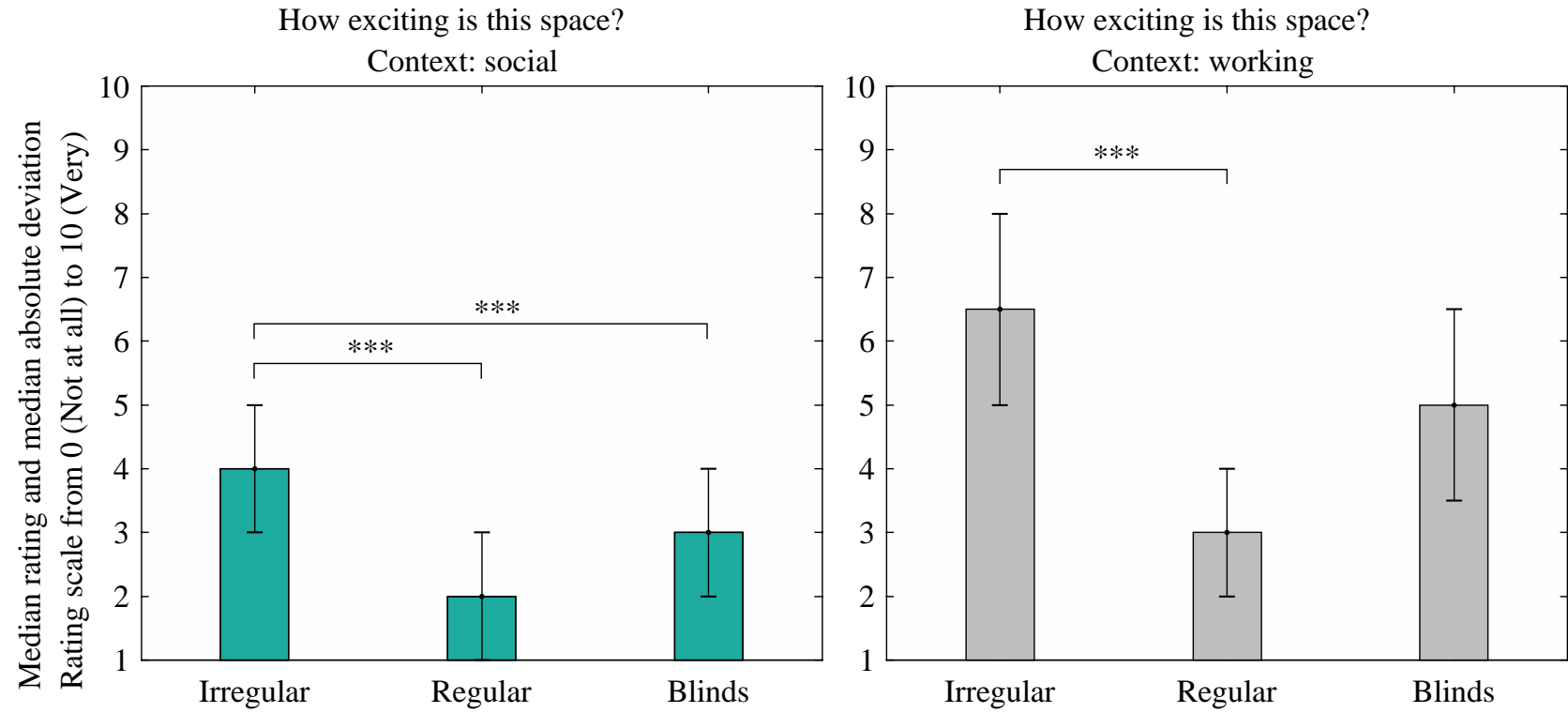

Figure 4. Median ratings and median absolute deviations for evaluations of how pleasant, interesting, and exciting the space was perceived under exposure to each façade geometry variation. In the case of a significant effect of context, the datasets of the two context scenarios are treated separately. 
The results show that the Irregular variation was evaluated as significantly more interesting and exciting than the Regular in both context scenarios $\left(\mathrm{W}_{\text {social }}=41, \mathrm{p}_{\text {social }}<.001, \mathrm{r}_{\text {social }}=.43, \mathrm{~W}_{\text {work }}=-\right.$ $4.10, \mathrm{p}_{\mathrm{work}}<.001, \mathrm{r}_{\mathrm{work}}=.48$, excitement: $\mathrm{W}_{\text {social }}=34.5, \mathrm{p}_{\text {social }}<.001, \mathrm{r}_{\text {social }}=.46, \mathrm{~W}_{\text {work }}=-3.93, \mathrm{p}_{\text {work }}$ $\left.<.001, \mathrm{r}_{\text {work }}=0.46\right)$, as well as more pleasant $(\mathrm{W}=263.5, \mathrm{p}<.001, \mathrm{r}=.41)$. The consistent difference between the Irregular and Regular condition across subjective attributes and context scenarios, shown in Figure 4, clearly demonstrates that the spatial distribution of the façade openings and the resulting spatial distribution of the associated sunlight pattern is a defining element in how the space is perceived.

The Irregular variation was also evaluated as significantly more interesting and exciting than the Blinds, solely in the case of social context (interest: $\mathrm{W}_{\text {social }}=6, \mathrm{p}_{\text {social }}<.001, \mathrm{r}_{\text {social }}=.57$, excitement: $\left.\mathrm{W}_{\text {social }}=60, \mathrm{p}_{\text {social }}<.001, \mathrm{r}_{\text {social }}=.39\right)$, which supports the argument that it might be more appropriate than venetian blinds for non-working environments. Lastly, the Blinds variation was evaluated as more pleasant than the Regular $(\mathrm{W}=1454, \mathrm{p}<.001, \mathrm{r}=.34)$, as shown in Figure 4.

\subsection{Physiological responses}

The analysis of the effect of context on the physiological responses in each presented façade variation with a Wilcoxon Rank-Sum test showed no significant differences between $\Delta H R$ responses in the two context scenarios (all ps>.08). The context scenario significantly affected the $\triangle \mathrm{SCR}$ in the Blinds $($ rank sum $=970.5, \mathrm{p}<.05, \mathrm{r}=.30)$ and Regular variations (rank sum $=1000, \mathrm{p}<.01, \mathrm{r}=.36)$. Following these results, ensuing analyses for $\Delta \mathrm{HR}$ were performed using the data from both contexts, while for $\Delta \mathrm{SCR}$, separate analyses were conducted for each context scenario.

A one-way Friedman's ANOVA for the three façade variations showed a statistically significant effect of pattern geometry on $\Delta \mathrm{HR}\left(\chi^{2}(2)=7.28, \mathrm{p}<.05\right)$, and no effect on $\Delta \mathrm{SCR}$ for either context scenario. This result confirms our second hypothesis that the joint impact of façade and sunlight pattern can affect the physiological responses of participants, but only in the case of heart rate responses. Post-hoc analysis on the $\Delta \mathrm{HR}$ with a Wilcoxon Signed-Ranks Matched-Pairs test 
showed significant differences between the Irregular and the Blinds variations $(\mathrm{W}=1177, \mathrm{p}<.05$, $\mathrm{r}=$.23), shown in Figure 5. Following the argument that cardiac deceleration indicates allocation of attentional resources to a perceived stimulus [43], this finding suggests that the participants' attention was higher towards the irregular façade geometry compared to the venetian blinds, and is conducive to our third hypothesis that the non-uniform distribution of openings will result to impressions of interest.

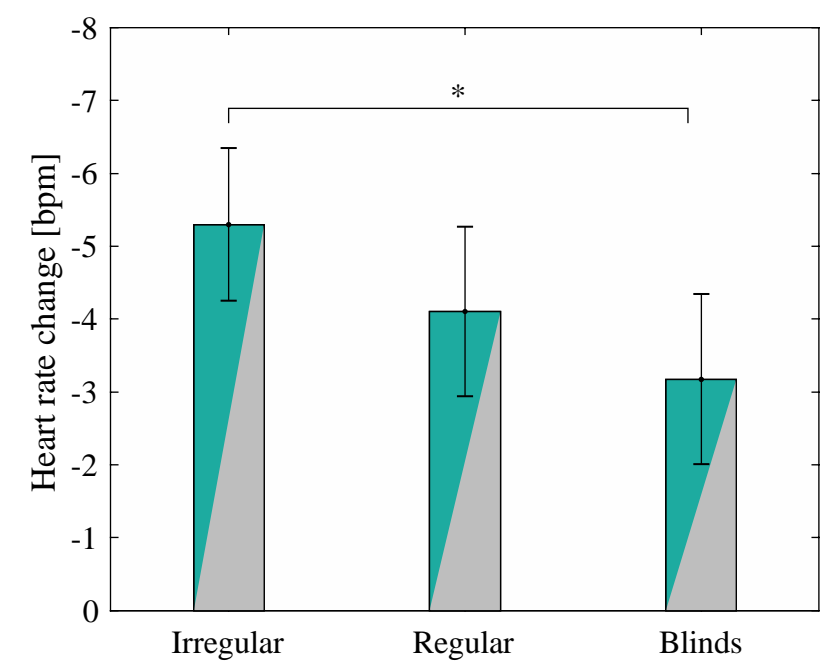

Dataset used:

$\square$ Social and working context $(\mathrm{N}=58)$

Significance level:

$*=\mathrm{p}<.05$

Figure 5. $\Delta \mathrm{HR}$ (calculated as the difference from the baseline) and standard error of the $\Delta \mathrm{HR}$ for a 28 s response window after onset of exposure to each façade variation, measured in bpm (beats per minute).

\subsection{Correlation between subjective and physiological responses}

It is of interest to investigate the correlation between the participants' subjective and physiological responses. In the following analyses, only a subset of the subjective responses is used, corresponding to the sample size of 58 participants that were used in the analysis of the physiological responses. Due the finding of a significant effect of context on perceived interest and perceived pleasantness, analyses are performed separately both for each context scenario and for each subjective attribute. To account for the multiple comparisons, we use a more conservative threshold for statistical significance with a Bonferroni correction and dividing the conventional significance level $\alpha$ of 0.05 by the number of analyses, $\alpha=.05 / 5=.01$.

The calculation of the Spearman's rho coefficient showed a statistically significant negative correlation between $\Delta \mathrm{HR}$ and reported interest in the case of social context (rho $=-0.45, \mathrm{p}<.0001$ ), 
which can interpreted as a small-to-moderate correlation [80]. No statistically significant correlation was found between $\Delta H R$ and excitement in the social context, between $\Delta H R$ and any of the perceptual impressions for the working context, or between $\Delta \mathrm{HR}$ and pleasantness (all ps<.04). The conduction of the same analysis for $\triangle \mathrm{SCR}$ showed no statistically significant correlation with subjective evaluations (all ps<.31).

\subsection{Effects of confounding variables}

In this section, we investigate possible effects of confounding variables on the findings of the study. To this end, we perform analyses on the effect of stimulus presentation order, time of day, and participant gender on all the studied variables for the three façade variations considered together. For brevity, only significant results are reported for this analysis. In addition, to address the air temperature variation in the experimental room, the data for all dependent variables was tested by comparing the two main temperature groups to ensure there is no effect of temperature. Results of a Wilcoxon Rank-Sum test revealed no significant effect of temperature for any of the studied variables.

The effect of stimulus presentation order (with six possible combinations) was investigated with a Kruskal-Wallis test, and showed no influence on any of the dependent variables. A Wilcoxon Rank-Sum test for the time of day of the experimental session (morning or afternoon) showed a significant effect of time on evaluations of pleasantness (rank sum $=12145, \mathrm{p}<.05, \mathrm{r}=.14$ ) and interest (rank sum $=12677, \mathrm{p}<0.01, \mathrm{r}=.22)$. The median ratings for the morning and afternoon sessions reveal that evaluations were more positive in the morning sessions for both how pleasant $\left(\right.$ median $_{\text {morning }}=5$, median $\left._{\text {afternoon }}=4\right)$ and how interesting $\left(\right.$ median $_{\text {morning }}=5$, median $\left._{\text {afternoon }}=4\right)$ the space was perceived, indicating perhaps that fatigue influenced the participants' judgements.

The effect of gender was investigated with a Wilcoxon Rank-Sum test. Gender was shown to have a significant effect on how exciting the space was perceived (rank sum $=10167, \mathrm{p}<.5$, rank sum $=1.0167 \mathrm{e}+04, \mathrm{r}=.18)$, as well as on $\Delta \mathrm{HR}($ rank sum $=8576, \mathrm{p}<.001, \mathrm{r}=.28)$ and $\Delta \mathrm{SCR}($ rank sum $=$ $6261, \mathrm{p}<.01, \mathrm{r}=0.25)$. In particular, men participants evaluated the space as more exciting $\left(\operatorname{median}_{\text {male }}\right.$ $=4$, median $\left._{\text {female }}=3\right)$ and they showed a response of larger magnitude on both $\Delta \mathrm{HR}\left(\right.$ mean $_{\text {male }}=-$ 
6.61, mean $\left._{\text {female }}=-1.61\right)$ and $\triangle S C R\left(\right.$ mean $_{\text {male }}=0.04$, mean $\left._{\text {female }}=-0.02\right)$. Although these findings do not affect the validity of the main results of this study, where gender was counterbalanced, they have important implications for the experimental design of future studies investigating these variables.

\section{Discussion}

This study introduces a novel experimental method that combines the projection of physically-based renderings from Radiance in virtual reality with the use of a wearable biometric sensor to investigate the joint impact of façade geometry and associated sunlight pattern on occupants. The findings demonstrate the potential of this method for empirical research on human perception, and highlight its usefulness as a promising experimental tool that can provide control of the visual stimuli and data collection of objective physiological indicators. Furthermore, this work extends our knowledge on the effects of daylight on occupants by showing both the impact of façade and sunlight patterns on subjective impressions and heart rate, and the importance of the use of space on occupant perception of luminous conditions. The presented results demonstrate in a virtual reality setting that architectural façade elements and the spatial distribution of sunlight can be used to impart specific sensations in the built environment. The following subsections discuss the findings regarding the joint effect of façade and sunlight pattern (referred to as "façade variation" for brevity) and the context scenario on the dependent variables of the study, the limitations of the current research, as well as directions of future work.

\subsection{Effect of façade variation and context scenario on subjective responses}

The influence of space use on perception was examined by employing two different context scenarios -socializing and working- of the presented virtual scene. Results showed that the context scenario had a significant effect on participants' evaluations of how interesting and exciting they perceived the space, supporting our fourth hypothesis that the participants' perception of the space will be influenced by the type of activity they expect to conduct in the presented scene. Furthermore, statistical analysis showed that the façade and sunlight pattern geometry significantly influenced the 
participants' ratings regarding how pleasant, interesting, and exciting the space is perceived for both context scenarios, which confirms our first hypothesis.

Following the aforementioned effects of façade variation on perception, we can use the median ratings to compare the direction of the evaluations, particularly in paired comparisons between the Irregular and Blinds, or the Irregular and Regular variations, which were often found to have statistically significantly differences. From Figure 4, we can observe that the Irregular variation was perceived as the most pleasant, interesting, and exciting within each context scenario, with the exception of the evaluations of pleasantness for the working context, where Blinds was the variation rated as most pleasant. The median ratings of excitement and interest for the Blinds in the two context scenarios, shown in Figure 4, reveal that this façade variation was rated more negatively in the context of a social space. While these finding are positive for common applications of venetian blinds in office spaces, they simultaneously bring into question the appropriateness of using venetian blinds in non-working environments where impressions of interest and excitement are desirable.

At the same time, Figure 4 shows that the Regular variation was rated as the least pleasant, interesting, and exciting. These observations, along with the significant differences between the evaluations of the Irregular and the Regular variations across all studied perceptual attributes, reveal that the spatial distribution of the apertures - the only feature that differed between these two façade geometry variations - has a direct impact on occupant perception. Furthermore, these findings confirm our third hypothesis that the non-uniformity in the case of the Irregular façade variation and the resulting sunlight pattern is inducive to impressions of interest, enhancing how interesting and exciting the space was perceived compared to the other studied façade geometry variations. These findings are in line with previous work by the authors [24], but differ from the work of Abboushi et al. [82], where the ratings of visual interest of horizontal stripes and fractal patterns applied to the glazing of a real office environment did not differ significantly between them. This outcome could be explained by the fact that in Abboushi et al. the shading system covered only a small part of the 
glazing, while in the present study it covered the whole window, and possibly had a greater effect on participant perception.

\subsection{Effect of façade variation and context scenario on physiological responses}

Context scenario had a significant effect on mean SCR change, but not on mean HR change. Further analyses revealed a significant effect of façade variation on mean HR change, which supports - only for the case of heart rate response - our second hypothesis that the joint impact of façade and sunlight pattern can influence the participants' physiological responses. In particular, during exposure to the scene with the Irregular variation, participants had a larger decrease in mean HR compared to the Blinds variation, as shown in Figure 5, which may suggest a coherent orienting effect [83], [52] towards this condition. This results suggests that the participants' attention was higher towards the Irregular variation compared to the Blinds, and supports our third hypothesis that the non-uniform distribution of openings will trigger impressions of interest in the scene.

The decrease in mean HR could also be due to a larger parasympathetic nervous system control over the cardiac output [84], which suggests that some conditions lead to improved recovery from what could be a mildly stressful situation in the baseline condition [85], since participants were uncertain of what was going to happen in the beginning of the experiment. An increased parasympathetic control over the cardiac output can be due to a more relaxed state or to less influence of the sympathetic branch (which activates due to arousing situations, putting the person in a state of alert [86]), which could also signify that the Irregular condition with the larger decrease in HR was more relaxing compared to the other conditions.

The finding of a statistically significant negative correlation between evaluations of interest and $\Delta \mathrm{HR}$ in the case of a social context scenario supports the argument that the focus of the participant's attention - which is also reflected in impressions of interest - is accompanied by cardiac deceleration [43]. However, this correlation was found only for the social context, which could be explained by the presence of a similar trend - in opposite direction - of a significant difference between the Irregular and the Blinds variations for both the perceived interest in that context and the mean heart 
rate response, which is not found in the case of the working context. The absence of correlation between $\triangle H R$ and the evaluations of how exciting the space is perceived indicates that it is not necessarily the same people that show a change in their HR response and evaluate the visual stimulus as exciting. This finding shows that the two measures are quite independent and the conjunction of these two cannot be discussed. A possible interpretation is that the question of "how exciting is the space" (labeled 'excitement' in the manuscript for ease) does not necessarily represent the participants' feelings of excitement (i.e. if the participant feels excited). This phrasing of the question was chosen according to the principle of atmosphere perception, which does not represent an affective state, but rather the participant's experience of the environment and is considered a more stable variable [87].

\subsection{Limitations}

This experimental study has demonstrated the joint impact of façade and sunlight pattern geometry on participant perception and HR through experiments in VR. These findings have important implications for architectural and lighting design, as they show that short exposure to joint façade and sunlight conditions could trigger specific subjective and physiological responses, and could thus be orchestrated in transition spaces, such as entrance or circulation zones, to achieve specific effects on occupants. However, it is important to note the limitations of the conducted experiment.

Due to the use of a VR headset, participants were exposed to each façade variation for a short period - with a mean exposure time of 68 seconds - which restricts the generalizability of the results. The outcomes of the study could be applied to settings where occupants spend an equivalent amount of time, such as transitional spaces, however further research is needed to investigate their applicability for longer exposure times. In the same vein, the participants' age was limited to avoid age-related effects. Although the use of a narrow age range ensures a more homogeneous population, it simultaneously requires the repetition of this investigation for other age groups. Regarding the influence of spatial context on the perception of luminous conditions, the use of context scenarios, where the participant is asked to imagine the use of the space, are inferior to actual variations of the 
furniture in the room, or to the involvement of the participant in different activities. Furthermore, no instructions were given to the participants to remain silent during recording of physiological measures, which might have influenced the recorded responses. Lastly, the use of a VR headset, while it allows identical repetition of the visual stimulus, has the disadvantage of a limited luminance range which cannot induce discomfort. Although in this study we successfully examine the impact of the spatial distribution of sunlight patterns on perception, we are restricted in investigating the threshold between interest and discomfort, which would be possible with luminance ranges close to those of a real environment.

\subsection{Future work}

One of the main findings of this study is that exposure to the façade variation with an irregular distribution of openings led to participants evaluating the space as more exciting and more interesting, as well as to cardiac deceleration, which has been suggested as a proxy for attention to a stimulus. These results are in alignment with previous findings by the authors [24], and could relate to existing work in the literature which associates the luminance variation in the occupant's field of view with impressions of interest [11], [19], [20]. Further studies are encouraged to investigate the replicability of the findings, especially regarding the physiological measures. A wider use of complementary physiological indicators, such as the mean heart rate change, which in this study was shown to be sensitive enough to differentiate between façade and sunlight pattern variations, could help establish these measures as important tools in lighting research studies on perception. Future work by the authors aims to investigate a wider range of façade variations and examine the effect of specific characteristics of façade and sunlight pattern geometry, such as complexity, which has been suggested in the field of aesthetics as a factor which influences preference [88]-[91].

\section{Conclusion}

In this study, we investigated the joint impact of façade and sunlight pattern geometry on occupant perception and physiological responses by exposing 72 participants to VR scenes of the same interior under clear sky with three façade variations of an equal aperture ratio: an irregular distribution of 
openings, a regular distribution of openings, and venetian blinds. Moreover, we examined the effect of the use of space on occupant perception by employing two different scenarios of spatial contextsocializing and working - of the presented virtual scene. A novel experimental method, using physically based renderings shown in immersive VR, was coupled with a verbal questionnaire - how pleasant, interesting, and exciting the space was perceived - and an Empatica E4 wristband, which measured heart rate and skin conductance response.

Results show a statistically significant effect of the façade geometry and the resulting sunlight pattern on participants' evaluations for all three subjective attributes. In particular, the irregular façade geometry variation was consistently evaluated across context scenarios as more pleasant, more interesting and more exciting than the regular façade variation, demonstrating the influence of the spatial distribution of façade openings. Moreover, the façade and sunlight pattern geometry significantly influenced the participant's mean heart rate, but not their skin conductance response. Specifically, under exposure to the irregular façade and sunlight pattern, participants' mean heart rate was lower compared to the venetian blinds, which suggest that participants' attention was higher towards the irregular pattern. The two spatial context scenarios - working and socializing in the space - affected subjective evaluations of interest and excitement, but not of pleasantness: the irregular façade variation was perceived as significantly more interesting and exciting than the venetian blinds for the social context, but not for the working context. This result highlights the relevance of considering the use of space in façade and lighting design, while challenging the use of prevalent shading system designs - such as venetian blinds - in non-working environments. In cases where impressions of interest and excitement are of importance, our results suggest that a façade with openings that are not regularly distributed, along with the induced sunlight patterns, will be perceived more positively compared to venetian blinds.

Our findings demonstrate that architectural façade elements and their interaction with light have a quantifiable effect on people in a virtual reality setting. In particular, the significant influence of façade and sunlight pattern geometry on mean heart rate change has great implications for 
architecture, as it shows that a simple change of a shading system - from venetian blinds to an irregular distribution of openings - can induce physiological changes to the occupant, such as cardiac deceleration. This potential of physiological benefits for the occupant calls for further research with a human-centric perspective, employing knowledge from fields such as psychophysics and neuroscience, traditionally remote from architecture and lighting [92].

\section{Acknowledgements}

The authors would like to extend their thanks to the LESO laboratory for access to the experimental room, to Peter Hansen for his help in setting up the experiment, and to all the participants that took part in the experiment.

\section{Funding}

This work was funded by the École Polytechnique Fédérale de Lausanne (EPFL) and through a grant awarded by the Velux Stiftung Foundation in the framework of Project 1022: Identifying the Impact of Regional Differences on the Perceived Quality of Daylit Architectural Spaces: A Comparison Study across Different Latitudes.”

\section{Declarations of interest: None}

\section{References}

[1] P. Zumthor, Atmospheres: architectural environments - surrounding objects. Basel: Birkhäuser, 2006.

[2] S. Holl, S. Kwinter, and J. Safont-Tria, Color, Light, Time, 1 edition. Zurich, Switzerland: Müller, Lars, 2011.

[3] J. Pallasmaa, The Eyes of the Skin: Architecture and the Senses, 3 edition. Chichester: Wiley, 2012.

[4] K. Steemers and M. A. Steane, Environmental Diversity in Architecture. London and New York: Routledge, 2012.

[5] IESNA, IES LM- 83-12, Approved Method: IES Spatial Daylight Autonomy (sDA) and Annual Sunlight Exposure (ASE). New York, NY, USA, 2012.

[6] J. Mardaljevic and A. Nabil, "The Useful Daylight Illuminance Paradigm: A Replacement for Daylight Factors," Light. Res. Technol., vol. 37, pp. 41-59, 2005.

[7] European Committee for Standardization CEN, "Revision of draft Standard prEN17037:2017, Daylight in buildings." 2017.

[8] H. Köster, Dynamic Daylighting Architecture: Basics, Systems, Projects. Basel, Boston, Berlin: Birkhäuser, 2004.

[9] M. Corrodi and K. Spechtenhauser, Illuminating: natural light in residential architecture. Basel, Boston, Berlin: Birkhäuser, 2008.

[10] J. A. Veitch, "Psychological Processes Influencing Lighting Quality," J. Illum. Eng. Soc., vol. 30, no. 1, pp. 124-140, Jan. 2001. 
[11] S. Rockcastle, M. L. Amundadottir, and M. Andersen, "Contrast measures for predicting perceptual effects of daylight in architectural renderings," Light. Res. Technol., vol. 49, no. 7, pp. 882-903, Apr. 2017.

[12] D. L. Butler and P. M. Biner, "Preferred Lighting Levels Variability among Settings, Behaviors, and Individuals," Environ. Behav., vol. 19, no. 6, pp. 695-721, Nov. 1987.

[13] N. Wang and M. Boubekri, "Investigation of declared seating preference and measured cognitive performance in a sunlit room," J. Environ. Psychol., vol. 30, no. 2, pp. 226-238, Jun. 2010.

[14] P. R. Boyce, Human Factors in Lighting, Third Edition. CRC Press, 2014.

[15] M. Boubekri, R. B. Hull, and L. L. Boyer, "Impact of Window Size and Sunlight Penetration on Office Workers' Mood and Satisfaction A Novel Way of Assessing Sunlight," Environ. Behav., vol. 23, no. 4, pp. 474-493, Jul. 1991.

[16] P. Leather, M. Pyrgas, D. Beale, and C. Lawrence, "Windows in the Workplace Sunlight, View, and Occupational Stress," Environ. Behav., vol. 30, no. 6, pp. 739-762, Nov. 1998.

[17] M. Boubekri and L. L. Boyer, "Effect of window size and sunlight presence on glare," Light. Res. Technol., vol. 24, no. 2, pp. 69-74, Jun. 1992.

[18] K. V. D. Wymelenberg, M. Inanici, and P. Johnson, "The Effect of Luminance Distribution Patterns on Occupant Preference in a Daylit Office Environment," LEUKOS, vol. 7, no. 2, pp. 103-122, Oct. 2010.

[19] D. L. Loe, K. P. Mansfield, and E. Rowlands, "Appearance of lit environment and its relevance in lighting design: Experimental study," Light. Res. Technol., vol. 26, no. 3, 1994.

[20] K. Parpairi, N. V. Baker, K. A. Steemers, and R. Compagnon, "The Luminance Differences index: a new indicator of user preferences in daylit spaces," Light. Res. Technol., vol. 34, no. 1, pp. 53-66, Mar. 2002.

[21] S. Rockcastle, K. Chamilothori, and M. Andersen, "Using Virtual Reality to Measure Daylight-Driven Interest in Rendered Architectural Scenes," in Proceedings of Building Simulation 2017, San Francisco, CA, USA, 2017.

[22] B. Abboushi and I. Elzeyadi, "Do visually interesting sunlight patterns impact occupants' perceived glare?," in IES Research Symposium 2018, Atlanta, GA, USA, 2018, p. 11.

[23] A. Omidfar, M. Niermann, and L. N. Groat, "The use of environmental aesthetics in subjective evaluation of daylight quality in office buildings," in Proceedings of IES Annual Conference, Indianapolis, 2015.

[24] K. Chamilothori, J. Wienold, and M. Andersen, "Daylight patterns as a means to influence the spatial ambiance: a preliminary study," in Proceedings of the 3rd International Congress on Ambiances, Volos, Greece, 2016.

[25] H. Bülow-Hübe, "Subjective reactions to daylight in rooms: Effect of using low-emittance coatings on windows," Light. Res. Technol., vol. 27, no. 1, pp. 37-44, Mar. 1995.

[26] G. R. Newsham, D. Cetegen, J. A. Veitch, and L. Whitehead, "Comparing lighting quality evaluations of real scenes with those from high dynamic range and conventional images," ACM Trans. Appl. Percept., vol. 7, no. 2, pp. 1-26, 2010.

[27] C. Hendrick, O. Martyniuk, T. J. Spencer, and J. E. Flynn, "Procedures for Investigating the Effect of Light on Impression Simulation of a Real Space by Slides," Environ. Behav., vol. 9, no. 4, pp. 491-510, Dec. 1977.

[28] C. Moscoso, B. Matusiak, U. P. Svensson, and K. Orleanski, "Analysis of Stereoscopic Images as a New Method for Daylighting Studies," ACM Trans. Appl. Percept. TAP, vol. 11, no. 4, pp. 21:1-21:13, Jan. 2015.

[29] A. Mahdavi and H. Eissa, "Subjective Evaluation of Architectural Lighting via Computationally Rendered Images," J. Illum. Eng. Soc., vol. 31, no. 2, pp. 11-20, Jul. 2002.

[30] G. R. Newsham, C. Richardson, C. Blanchet, and J. A. Veitch, "Lighting quality research using rendered images of offices," Light. Res. Technol., vol. 37, no. 2, pp. 93-115, Jun. 2005.

[31] C. Cauwerts, "Influence of presentation modes on visual perceptions of daylit spaces (Doctoral Dissertation)," Université catholique de Louvain, Louvain-la-Neuve, Belgium, 2013.

[32] Y. A. De Kort, W. A. Ijsselsteijn, J. Kooijman, and Y. Schuurmans, "Virtual laboratories: Comparability of real and virtual environments for environmental psychology," Presence Teleoperators Virtual Environ., vol. 12, no. 4, pp. 360-373, 2003.

[33] S. F. Kuliga, T. Thrash, R. C. Dalton, and C. Hölscher, "Virtual reality as an empirical research tool Exploring user experience in a real building and a corresponding virtual model," Comput. Environ. Urban Syst., 2015.

[34] A. Heydarian, J. P. Carneiro, D. Gerber, B. Becerik-Gerber, T. Hayes, and W. Wood, "Immersive virtual environments versus physical built environments: A benchmarking study for building design and userbuilt environment explorations," Autom. Constr., vol. 54, pp. 116-126, Jun. 2015. 
[35] K. Chamilothori, J. Wienold, and M. Andersen, "Adequacy of Immersive Virtual Reality for the Perception of Daylit Spaces: Comparison of Real and Virtual Environments," LEUKOS, pp. 1-24, Jan. 2018.

[36] J. A. Russell, "A circumplex model of affect," J. Pers. Soc. Psychol., vol. 39, no. 6, pp. 1161-1178, 1980.

[37] J. A. Russell, L. M. Ward, and G. Pratt, "Affective Quality Attributed to Environments: A Factor Analytic Study," Environ. Behav., vol. 13, no. 3, pp. 259-288, May 1981.

[38] J. R. Fontaine, K. R. Scherer, E. B. Roesch, and P. C. Ellsworth, "The world of emotions is not twodimensional," Psychol. Sci., vol. 18, no. 12, pp. 1050-1057, 2007.

[39] K. W. Houser and D. K. Tiller, "Measuring the subjective response to interior lighting: paired comparisons and semantic differential scaling," Light. Res. Technol., vol. 35, no. 3, pp. 183-195, Sep. 2003.

[40] M. Stokkermans, I. Vogels, Y. de Kort, and I. Heynderickx, "A Comparison of Methodologies to Investigate the Influence of Light on the Atmosphere of a Space," LEUKOS, vol. 0, no. 0, pp. 1-25, Nov. 2017.

[41] D. K. Tiller and M. S. Rea, "Semantic differential scaling: Prospects in lighting research," Light. Res. Technol., vol. 24, no. 1, pp. 43-51, Mar. 1992.

[42] M. E. Dawson, A. M. Schell, and D. L. Filion, "The Electrodermal System," in Handbook of Psychophysiology, Third edition., J. T. Cacioppo, G. G. Berntson, and L. G. Tassinary, Eds. Cambridge University Press, 2007, pp. 200-223.

[43] J. I. Lacey and B. C. Lacey, "Verification and Extension of the Principle of Autonomic ResponseStereotypy," Am. J. Psychol., vol. 71, no. 1, p. 50, Mar. 1958.

[44] L. Izso, E. Lang, L. Laufer, S. Suplicz, and A. Horvath, "Psychophysiological, performance and subjective correlates of different lighting conditions," Light. Res. Technol., vol. 41, no. 4, pp. 349-360, Dec. 2009.

[45] S. D. Kreibig, "Autonomic nervous system activity in emotion: A review," Biol. Psychol., vol. 84, no. 3, pp. 394-421, Jul. 2010.

[46] P. J. Lang, M. K. Greenwald, M. M. Bradley, and A. O. Hamm, "Looking at pictures: affective, facial, visceral, and behavioral reactions," Psychophysiology, vol. 30, no. 3, pp. 261-273, May 1993.

[47] P. J. Lang, "The emotion probe. Studies of motivation and attention," Am. Psychol., vol. 50, no. 5, pp. 372-385, 1995.

[48] E. Bernat, C. J. Patrick, S. D. Benning, and A. Tellegen, "Effects of picture content and intensity on affective physiological response," Psychophysiology, vol. 43, no. 1, pp. 93-103, Jan. 2006.

[49] E. S. Dan-Glauser and K. R. Scherer, "The Geneva affective picture database (GAPED): a new 730picture database focusing on valence and normative significance," Behav. Res. Methods, vol. 43, no. 2, p. 468, Jun. 2011.

[50] M. Geiser and P. Walla, "Objective Measures of Emotion During Virtual Walks through Urban Environments," Appl. Sci., vol. 1, no. 1, pp. 1-11, Jul. 2011.

[51] A. Felnhofer et al., "Is virtual reality emotionally arousing? Investigating five emotion inducing virtual park scenarios," Int. J. Hum.-Comput. Stud., vol. 82, pp. 48-56, Oct. 2015.

[52] K. Laumann, T. Gärling, and K. M. Stormark, "Selective attention and heart rate responses to natural and urban environments," J. Environ. Psychol., vol. 23, no. 2, pp. 125-134, Jun. 2003.

[53] M. Inanici, "Evaluation of high dynamic range photography as a luminance data acquisition system," Light. Res. Technol., vol. 38, no. 2, pp. 123-136, Jun. 2006.

[54] E. Reinhard, W. Heidrich, P. Debevec, S. Pattanaik, G. Ward, and K. Myszkowski, High Dynamic Range Imaging: Acquisition, Display, and Image-Based Lighting. Morgan Kaufmann, 2010.

[55] E. Reinhard, M. Stark, P. Shirley, and J. Ferwerda, "Photographic Tone Reproduction for Digital Images," in Proceedings of the 29th Annual Conference on Computer Graphics and Interactive Techniques, New York, NY, USA, 2002, pp. 267-276.

[56] A. Lang, "Involuntary Attention and Physiological Arousal Evoked by Structural Features and Emotional Content in TV Commercials," Commun. Res., vol. 17, no. 3, pp. 275-299, Jun. 1990.

[57] A. Lang, S. Geiger, M. Strickwerda, and J. Sumner, "The Effects of Related and Unrelated Cuts on Television Viewers' Attention, Processing Capacity, and Memory," Commun. Res., vol. 20, no. 1, pp. 4-29, 1993.

[58] W. M. Winton, L. E. Putnam, and R. M. Krauss, "Facial and autonomic manifestations of the dimensional structure of emotion," J. Exp. Soc. Psychol., vol. 20, no. 3, pp. 195-216, May 1984. 
[59] N. Ravaja, T. Saari, M. Salminen, J. Laarni, and K. Kallinen, "Phasic Emotional Reactions to Video Game Events: A Psychophysiological Investigation," Media Psychol., vol. 8, no. 4, pp. 343-367, Nov. 2006.

[60] E. Bekele, Z. Zheng, A. Swanson, J. Crittendon, Z. Warren, and N. Sarkar, "Understanding How Adolescents with Autism Respond to Facial Expressions in Virtual Reality Environments," IEEE Trans. Vis. Comput. Graph., vol. 19, no. 4, Apr. 2013.

[61] M. Benedek and C. Kaernbach, "A continuous measure of phasic electrodermal activity," J. Neurosci. Methods, vol. 190, no. 1, pp. 80-91, Jun. 2010.

[62] Society for Psychophysiological Research Ad Hoc Committee on Electrodermal Measures et al., "Publication recommendations for electrodermal measurements," Psychophysiology, vol. 49, no. 8, pp. 1017-1034, Aug. 2012.

[63] A. Thanachareonkit and J.-L. Scartezzini, "Modelling Complex Fenestration Systems using physical and virtual models," Sol. Energy, vol. 84, no. 4, pp. 563-586, Apr. 2010.

[64] G. Ward Larson, "The RADIANCE lighting simulation and rendering system," in Proceedings of the 21 st annual conference on Computer graphics and interactive techniques, New York, NY, USA, 1994, pp. $459-472$.

[65] A. Jakubiec and C. F. Reinhart, "DIVA 2.0: integrating daylight and thermal simulations using Rhinoceros 3D, DAYSIM and EnergyPlus," in Proceedings of Building Simulation (IBPSA), Sidney, Australia, 2011, pp. 2202-2209.

[66] M. Mahy, L. V. Eycken, and A. Oosterlinck, "Evaluation of Uniform Color Spaces Developed after the Adoption of CIELAB and CIELUV," Color Res. Appl., vol. 19, no. 2, pp. 105-121, Apr. 1994.

[67] EN ISO, “12464-1-2011: Light and lighting-Lighting of work places-Part 1: indoor work places," CEN, Brussels, 2011.

[68] M. Garbarino, M. Lai, D. Bender, R. W. Picard, and S. Tognetti, "Empatica E3 \#x2014; A wearable wireless multi-sensor device for real-time computerized biofeedback and data acquisition," in 2014 4th International Conference on Wireless Mobile Communication and Healthcare - Transforming Healthcare Through Innovations in Mobile and Wireless Technologies (MOBIHEALTH), 2014, pp. 39 42.

[69] C. McCarthy, N. Pradhan, C. Redpath, and A. Adler, "Validation of the Empatica E4 wristband," in 2016 IEEE EMBS International Student Conference (ISC), 2016, pp. 1-4.

[70] J. L. Higuera-Trujillo, J. López-Tarruella Maldonado, and C. Llinares Millán, "Psychological and physiological human responses to simulated and real environments: A comparison between Photographs, $360^{\circ}$ Panoramas, and Virtual Reality," Appl. Ergon., vol. 65, pp. 398-409, Nov. 2017.

[71] J. Pietilä et al., "Evaluation of the accuracy and reliability for photoplethysmography based heart rate and beat-to-beat detection during daily activities," in EMBEC \& NBC 2017, 2018, pp. 145-148.

[72] M. Z. Poh, N. C. Swenson, and R. W. Picard, "A Wearable Sensor for Unobtrusive, Long-Term Assessment of Electrodermal Activity," IEEE Trans. Biomed. Eng., vol. 57, no. 5, pp. 1243-1252, May 2010.

[73] A. Kuijsters, J. Redi, B. de Ruyter, P. Seuntiëns, and I. Heynderickx, "Effects of Ageing on Atmosphere Perception," in Proceedings of Experiencing Light 2012, Eindhoven, The Netherlands, 2012, pp. 1-5.

[74] S. Schweitzer et al., "Investigation of gender- and age-related preferences of men and women regarding lighting conditions for activation and relaxation," presented at the SPIE Optical Engineering + Applications, San Diego, California, United States, 2016, p. 99540L.

[75] G. Labouvie-Vief, M. A. Lumley, E. Jain, and H. Heinze, "Age and gender differences in cardiac reactivity and subjective emotion responses to emotional autobiographical memories," Emot. Wash. DC, vol. 3, no. 2, pp. 115-126, Jun. 2003.

[76] D. P. Smith, C. H. Hillman, and A. R. Duley, "Influences of Age on Emotional Reactivity During Picture Processing," J. Gerontol. Ser. B, vol. 60, no. 1, pp. P49-P56, Jan. 2005.

[77] M. B. Neiss, L. A. Leigland, N. E. Carlson, and J. S. Janowsky, "Age differences in perception and awareness of emotion," Neurobiol. Aging, vol. 30, no. 8, pp. 1305-1313, Aug. 2009.

[78] S. Siegel, Nonparametric Statistics for the Behavioral Sciences, 1st edition. New-York: McGraw-Hill, 1956.

[79] R. Rosenthal, "Parametric measures of effect size," in The handbook of research synthesis, New York, NY, US: Russell Sage Foundation, 1994, pp. 231-244.

[80] C. J. Ferguson, "An effect size primer: A guide for clinicians and researchers.," Prof. Psychol. Res. Pract., vol. 40, no. 5, pp. 532-538, 2009. 
[81] C. Spearman, "The proof and measurement of association between two things.," Am. J. Psychol., vol. 100, no. 3-4, pp. 441-471, 1987.

[82] B. Abboushi and I. Elzeyadi, "The Relationship between Sunlight Pattern Geometry and Visual Comfort in Daylit Offices," in Proceedings of the ARCC Conference, Philadelphia, 2018, p. 9.

[83] F. K. Graham and R. K. Clifton, "Heart-rate change as a component of the orienting response.," Psychol. Bull., vol. 65, no. 5, pp. 305-320, 1966.

[84] J. Sztajzel, "Heart rate variability: a noninvasive electrocardiographic method to measure the autonomic nervous system.," Swiss Med. Wkly., vol. 134, no. 35-36, pp. 514-522, Sep. 2004.

[85] Y. M. Ulrich-Lai and J. P. Herman, "Neural regulation of endocrine and autonomic stress responses," Nat. Rev. Neurosci., vol. 10, no. 6, pp. 397-409, Jun. 2009.

[86] B. M. Appelhans and L. J. Luecken, "Heart rate variability as an index of regulated emotional responding," Rev. Gen. Psychol., vol. 10, no. 3, pp. 229-240, 2006.

[87] I. Vogels, "Atmosphere Metrics - Development of a Tool to Quantify Experienced Atmosphere," in Probing Experience: From Assessment of User Emotions and Behaviour to Development of Products, J. H. D. M. Westerink, Ed. Dordrecht, the Netherlands: Springer, 2008, pp. 25-41.

[88] T. Jacobsen and L. Höfel, "Aesthetic judgments of novel graphic patterns: analyses of individual judgments," Percept. Mot. Skills, vol. 95, no. 3 Pt 1, pp. 755-766, Dec. 2002.

[89] J. Friedenberg and B. Liby, "Perceived beauty of random texture patterns: A preference for complexity," Acta Psychol. (Amst.), vol. 168, no. Supplement C, pp. 41-49, Jul. 2016.

[90] A. Forsythe, M. Nadal, N. Sheehy, C. J. Cela-Conde, and M. Sawey, "Predicting beauty: Fractal dimension and visual complexity in art," Br. J. Psychol., vol. 102, no. 1, pp. 49-70, Feb. 2011.

[91] M. M. Marin and H. Leder, "Examining Complexity across Domains: Relating Subjective and Objective Measures of Affective Environmental Scenes, Paintings and Music," PLOS ONE, vol. 8, no. 8, p. e72412, Aug. 2013.

[92] M. Andersen, "Unweaving the human response in daylighting design," Build. Environ., vol. 91, pp. 101117, Sep. 2015.

\section{Appendix}

Table A. Verbal questionnaire

\begin{tabular}{ll}
\hline Pleasant & How pleasant is this space? \\
Interesting & I would like you to answer on a scale from 1 to 10,1 being "Not at all" and 10 being "Very". \\
& How interesting is this space? \\
Exciting & I would like you to answer on a scale from 1 to 10,1 being "Not at all" and 10 being "Very". \\
& How exciting is this space? \\
& I would like you to answer on a scale from 1 to 10,1 being "Not at all" and 10 being "Very".
\end{tabular}

Table B. Radiance material properties for the main surfaces

\begin{tabular}{llllllll}
\hline Surface & Type & $R$ & $G$ & $B$ & Reflectance & Specularity & Visual Transmittance \\
Ceiling & plastic & 0.93 & 0.92 & 0.86 & $92 \%$ & 0 & \\
Floor & plastic & 0.46 & 0.47 & 0.48 & $47 \%$ & 0 & \\
Walls & plastic & 0.92 & 0.92 & 0.89 & $92 \%$ & 0.01 & \\
Table & plastic & 0.75 & 0.75 & 0.73 & $75 \%$ & 0.01 & \\
Facade & plastic & 0.90 & 0.89 & 0.86 & $89 \%$ & 0 & $88 \%$ \\
Glazing* & glass & 0.96 & 0.96 & 0.96 & & & \\
*A non-coated double paned glazing was used for the window.
\end{tabular}

